\title{
Asymmetrical Flow Driving in Two-Sided Lid-Driven Square Cavity with Antiparallel Wall Motion
}

\author{
El Amin Azzouz ${ }^{1, *}$, Samir Houat ${ }^{2}$ \\ ${ }^{1}$ Institute of Maintenance and Industrial Safety, University Mohamed Ben Ahmed Oran2, Oran, Algeria. \\ ${ }^{2}$ MSMPT Group, MNEPM Laboratory, University of Abdelhamid IbnBadis of Mostaganem, Mostaganem, Algeria.
}

\begin{abstract}
The two-dimensional flow in a two-sided lid-driven cavity is often handled numerically for the same imposed wall velocities (symmetrical driving) either for parallel or antiparallel wall motion. However, in this study, we present a finite volume method (FVM) based on the second scheme of accuracy to numerically explore the steady two-dimensional flow in a two-sided lid-driven square cavity for antiparallel wall motion with different imposed wall velocities (asymmetrical driving). The top and the bottom walls of the cavity slide in opposite directions simultaneously at different velocities related to various imposed velocity ratios, $\lambda=-2,-6$, and -10 , while the two remaining vertical walls are stationary. The results show that varying the velocity ratio and consequently the Reynolds ratios have a significant effect on the flow structures and fluid properties inside the cavity.
\end{abstract}

\section{Introduction}

The two-sided lid-driven cavity problem has been well suited for benchmarking following the conventional onesided lid-driven cavity. Their type of flow bear similarities to a lot of engineering applications including coating and drying technologies, polymers melts, and chemical etching of film cooling.

It has been initiated both experimentally and numerically by Kuhlmann et al. [1, 2] in order to investigate two- and three-dimensional flow and elucidate the instability process in a two-sided lid-driven rectangular cavity.

Fruitful numerical results have been presented by Albensoeder et al. [3] to investigate multiple twodimensional steady flows in a two-sided lid-driven cavity with various aspect ratios and Reynolds numbers. They have identified the region of no uniqueness and the existence range of multiple solutions.

In the last few years, for the same imposed wall velocity (symmetrical driving), a set of numerical simulations has been recently carried out by [4-13] to examine a twodimensional steady flow in a two-sided lid-driven cavity with various aspect ratios either in parallel or antiparallel wall motion. Within the variety of the employed numerical codes, a great resemblance among all studies was found. Although Che-Sidik and Razali [14] have numerically explored the asymmetric fluid flow in two-sided lid-driven cavity induced due to various imposed wall velocities in parallel wall motion, to our knowledge, there is no published data for the predicted two-dimensional flow in two-sided lid driven-cavity with various imposed wall velocities in antiparallel wall motion. Thus, the present paper aims to examine steady two-dimensional asymmetrically induced flow in a two-sided lid-driven square cavity with antiparallel wall motion for various velocity ratios, $\lambda=-2,-6$, and -10 . The impact of velocity ratios on the arising flow patterns will be presented with the finite volume method (FVM).

\section{Problem statement and mathematical model}

A two-dimensional two-sided facing lid-driven square cavity filled with a fluid set into motion is considered (Figure1). The top wall moves in the right direction with different velocities, $U_{\mathrm{T}}=\lambda U_{\mathrm{B}}$, involving a variable velocity ratio, $\lambda=U_{\mathrm{T}} / U_{\mathrm{B}}$. The bottom wall moves to the left direction with a constant velocity $U_{\mathrm{B}}$ obtained through $R e_{2}=100$. The two remaining vertical walls are taken to be at rest.

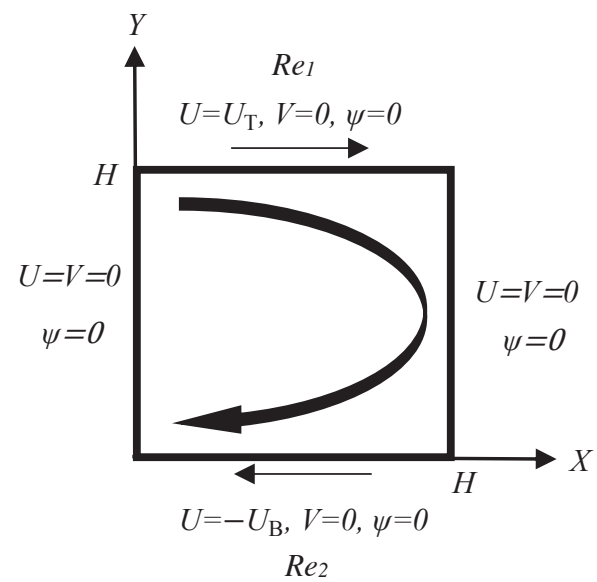

Fig. 1. Physical domain and boundary conditions.

\footnotetext{
* Corresponding author: aminazouz31@yahoo.com
} 
The fluid flow investigated in this work is assumed incompressible, Newtonian, and laminar. Dimensionless equations of mass and momentum can be written in the Cartesian coordinates for two-dimensional flow as follow:

$$
\begin{gathered}
\frac{\partial U}{\partial X}+\frac{\partial V}{\partial Y}=0 \\
U \frac{\partial U}{\partial X}+V \frac{\partial U}{\partial Y}=-\frac{\partial P}{\partial X}+\frac{1}{\operatorname{Re}}\left[\frac{\partial^{2} U}{\partial X^{2}}+\frac{\partial^{2} U}{\partial Y^{2}}\right] \\
U \frac{\partial V}{\partial X}+V \frac{\partial V}{\partial Y}=-\frac{\partial P}{\partial Y}+\frac{1}{\operatorname{Re}}\left[\frac{\partial^{2} V}{\partial X^{2}}+\frac{\partial^{2} V}{\partial Y^{2}}\right]
\end{gathered}
$$

Where $X, Y, U, V, P$ represent dimensionless Cartesian coordinates, fluid velocity components, and pressure, respectively. The Reynolds number can be written as:

$$
R e_{1,2}=\frac{\rho U_{T, B} H}{\mu}
$$

Where $H, \rho, \mu$ denote the physical size of the cavity, fluid density, and fluid dynamic viscosity, respectively. Velocity components could be obtained in terms of streamfunction $(\psi)$ and vorticity $(\omega)$ by:

$$
U=-\frac{\partial \psi}{\partial Y}, V=\frac{\partial \psi}{\partial X}, \nabla^{2} \psi=\omega
$$

\section{Method of solution}

To carry out the numerical analysis, a finite volume method [15] based on the second scheme of accuracy was employed to solve the transport equations presented by continuity Eq. (1), and momentum Eq. $(2,3)$. The simple algorithm Patankar et Spalding [16] is used to handle the pressure-velocity coupling. The convergence criteria adopted in this paper was based on a maximum residual of RES $=10^{-8}$ as a measure of confirmation of the convergence to the steady-state with very accurate solutions.

\section{Grid dependence and Code validation}

To select an optimum grid size with less computational time and good accuracy, a grid sensitivity analysis is carried out. The mid-plane $U$-velocity components are calculated for $\mathrm{Re}=1000$ with four cases of non-uniform grids: $81 \times 81,161 \times 161,257 \times 257$, and $513 \times 513$ as shown from Figure 2. It can be seen that the difference becomes marginal and the effect on the solutions accuracies is almost the same for the mesh sizes, $257 \times 257$, and $513 \times 513$. Hence, to save time consumption, A structured hexahedral mesh of $257 \times 257$ points distributed in a nonequal manner is adopted and used for all calculations. Then, the credibility of the present code is thought about. For this purpose, the present numerical results are compared with the popular one-sided lid-driven cavity data by Ghia et al. [17] obtained on a coarse grid of $129 \times 129$.
Figure 3 shows the comparison of the mid-plane $U$ components velocity for different Reynolds numbers. As clearly seen, a very good congruence between the two numerical results is obtained. Thereby, the present code stands validated.

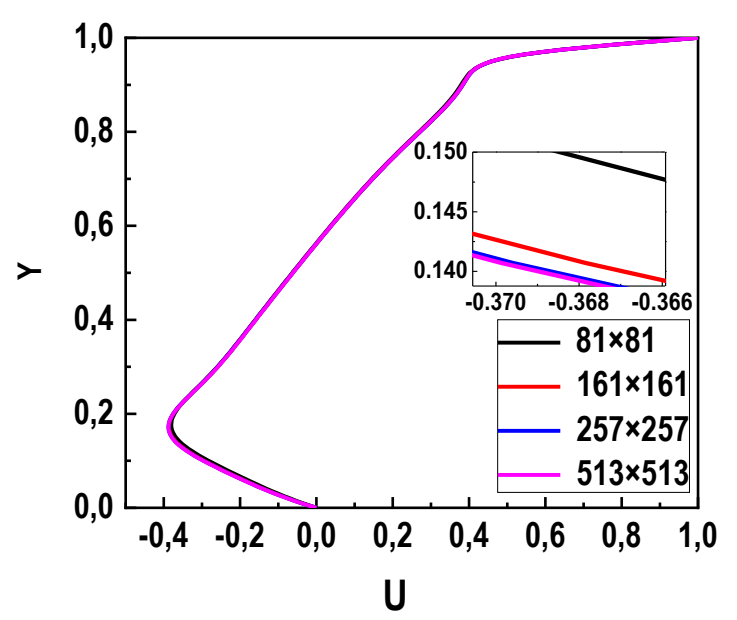

Fig. 2. Grid dependency test of $U$-velocity components passing through $X=1 / 2$ for $\mathrm{Re}=1000$ with various mesh resolution.

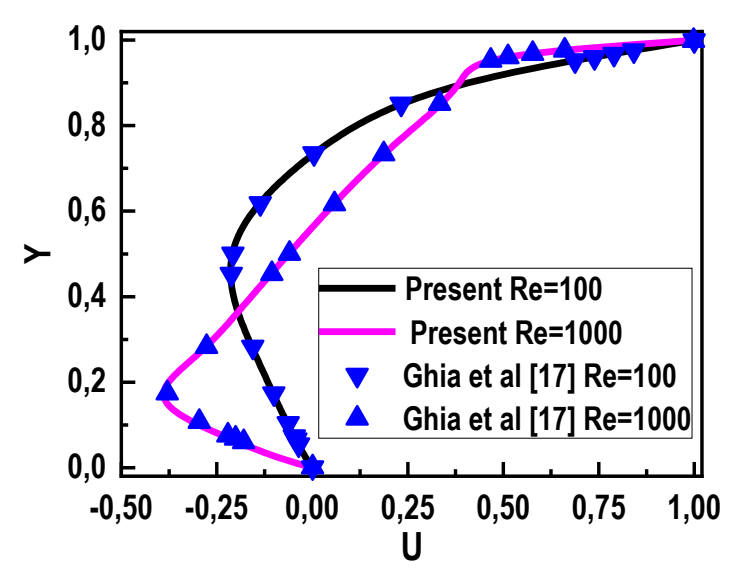

Fig. 3. Code validation, $U$-velocity components passing through $X=1 / 2$ for $\mathrm{Re}=100$ and $\mathrm{Re}=1000$ compared with results by Ghia et al [17].

\section{Results and discussions}

Steady two-dimensional asymmetrical flow driving in a two-sided lid-driven square cavity with antiparallel wall motion $\left(\operatorname{Re}_{1} \neq \operatorname{Re}_{2} ; U_{\mathrm{T}} \neq-U_{\mathrm{B}}\right)$ has been examined for various velocity ratios $(\lambda=-2,-6,-10)$. The detailed analysis of the flow results is presented in terms of stream functionvorticity contours, fluid proprieties, and velocity profiles. Figure 4 depicts stream-function contours obtained for $R e_{2}=100$ at various velocity ratios, $\lambda=-2,-6$, and -10 . It is obviously seen that the topology of the flow patterns inside the square cavity is affected by the augmentation of the absolute velocity ratios $(\lambda)$. Indeed, the flow is asymmetrically accelerated by a significant amount of motion by the top wall comparing to the bottom wall 
leading to break the point-symmetry about the centre of the cavity.

For $\lambda=-2$, the fluid flow consists of a clockwise rotating primary vortex occupying most of the cavity. The centre of this vortex found to be somewhat shifted from the centre of the cavity toward the top right corner.
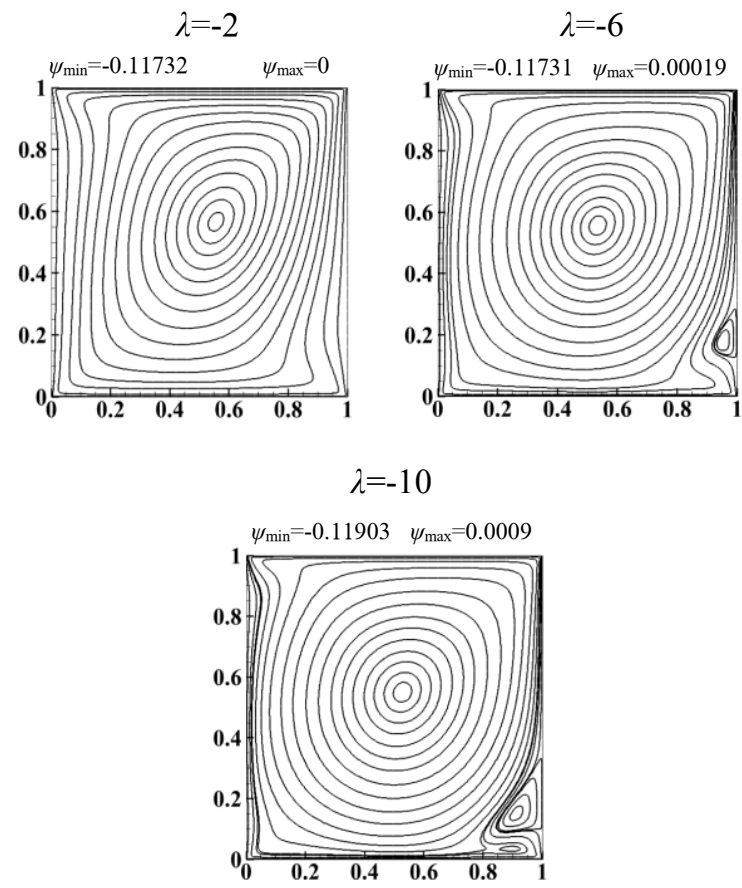

Fig. 4. Stream-function contours for various velocity ratios.
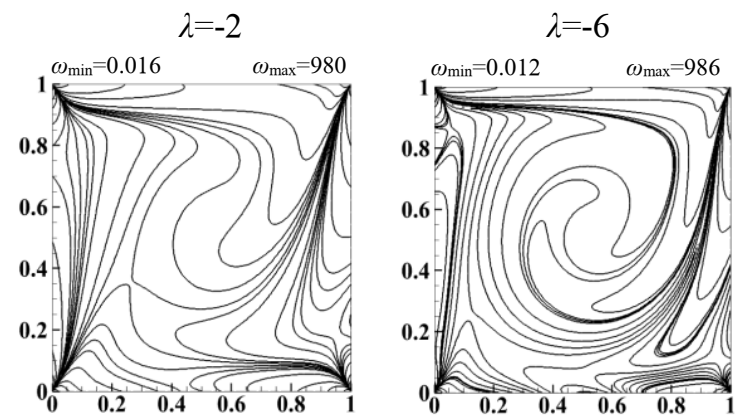

$$
\lambda=-10
$$

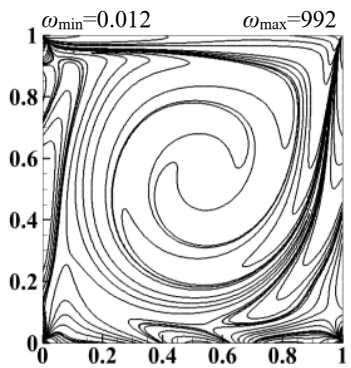

Fig. 5. Vorticity contours for various velocity ratios.

For $\lambda=-6$, in addition to the primary vortex, a small anticlockwise secondary vortex appears adjacent to the lower right corner which tends to grow in size and strength with the rising absolute velocity ratios.
For $\lambda=-10$, a gradual formation of a nearly stagnant region is observed beneath the minor secondary vortex. Expectedly, a second secondary vortex starts to appear in this region with a clockwise rotating. Whereas, the primary vortex centre is seen to be shifted gradually towards the lower-left direction to be somewhat near the geometric centre of the cavity.

Figure 5 shows vorticity contours for $\lambda=-2,-6$, and -10 where high gradients of vorticity can be distinguished by concentrations.

Table 1. Locations of primary and secondary vortices centre for various velocity ratios.

\begin{tabular}{|c|c|c|c|c|}
\hline \multirow{2}{*}{$\lambda$} & \multicolumn{2}{|c|}{ Primary vortex } & \multicolumn{2}{c|}{$\begin{array}{c}\text { First bottom right } \\
\text { secondary vortex }\end{array}$} \\
\cline { 2 - 5 } & $X$ & $Y$ & $X$ & $Y$ \\
\hline-2 & 0.55595 & 0.56593 & - & - \\
\hline-6 & 0.53415 & 0.55663 & 0.95140 & 0.17800 \\
\hline-10 & 0.52767 & 0.55053 & 0.91200 & 0.14995 \\
\hline
\end{tabular}

Table 2. Values of stream-function and vorticity for primary and secondary vortices at various velocity ratios.

\begin{tabular}{|c|c|c|c|c|}
\hline \multirow{2}{*}{$\lambda$} & \multicolumn{2}{|c|}{ Primary vortex } & \multicolumn{2}{c|}{$\begin{array}{c}\text { Bottom right secondary } \\
\text { vortex }\end{array}$} \\
\cline { 2 - 5 } & $\psi$ & $\omega$ & $\psi$ & $\omega$ \\
\hline-2 & -0.11732 & -2.11583 & - & - \\
\hline-6 & -0.11730 & -2.03257 & $1.98922 \mathrm{E}-04$ & 0.64683 \\
\hline-10 & -0.11902 & -1.99884 & $9.48230 \mathrm{E}-04$ & 1.22412 \\
\hline
\end{tabular}

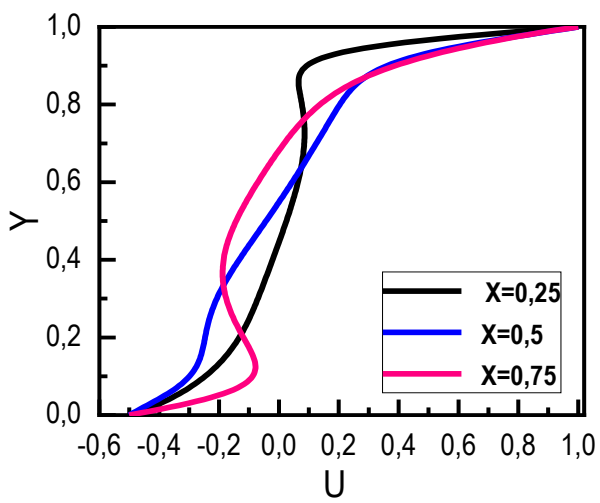

Fig. 6. Horizontal velocity Profile $U$ for $\lambda=-2$ through the vertical lines $(X=0.25, X=0.5, X=0.75)$.

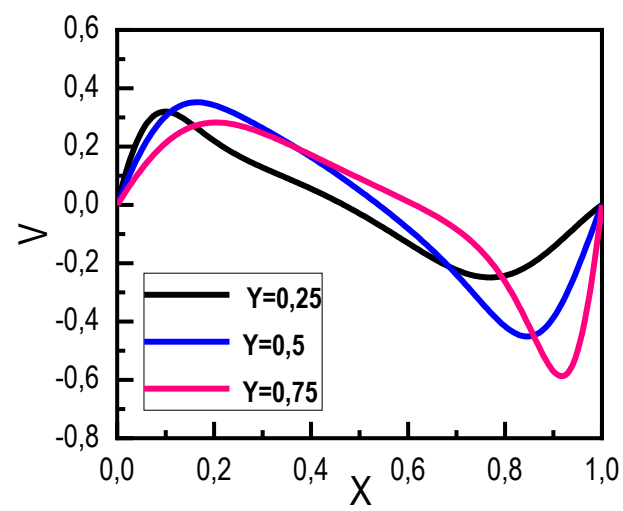

Fig. 7. Vertical velocity profile $V$ for $\lambda=-2$ through the horizontal lines $(Y=0.25, Y=0.5, Y=0.75)$. 


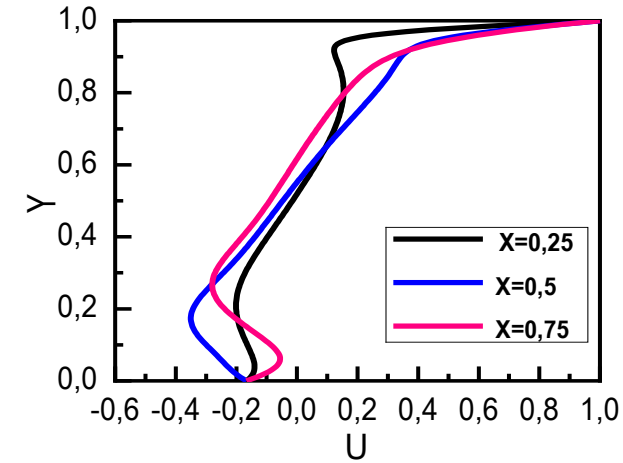

Fig. 8. Horizontal velocity Profile $U$ for $\lambda=-6$ through the vertical lines $(X=0.25, X=0.5, X=0.75)$.

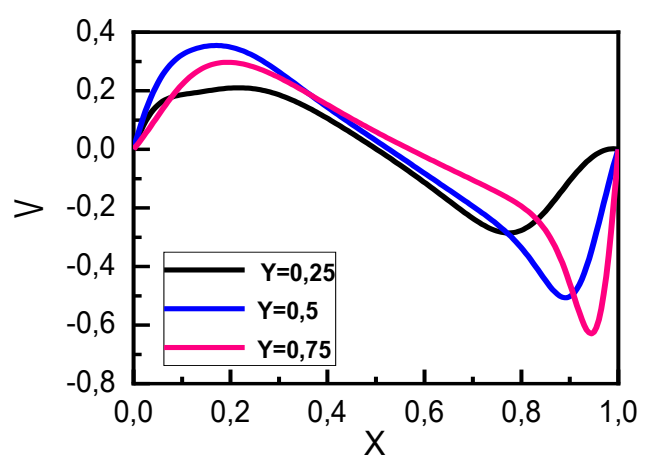

Fig. 9. Vertical velocity profile $V$ for $\lambda=-6$ through the horizontal lines $(Y=0.25, Y=0.5, Y=0.75)$.

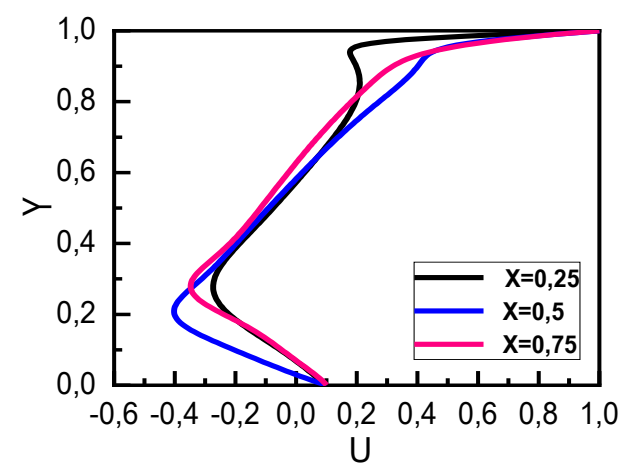

Fig. 10. Horizontal velocity Profile $U$ for $\lambda=-10$ through the vertical lines $(X=0.25, X=0.5, X=0.75)$.

Tables 1, 2 respectively show the locations centre and fluid properties in term of stream-function and vorticity values for primary and secondary vortices when $R e_{2}=100$ and $\lambda=-$ 2 , -6 , and -10 . These benchmarking results reflect the physics of the flow inside the cavity which has been investigated herein.

The effect of various velocity ratios can be better understood by examining the velocity profiles inside the cavity. Figures 6, 8, 10 and Figures 7, 9, 11 show respectively the $U$ - and $V$-velocities components along the vertical lines $(X=0.25,0.5,0.75)$ and the horizontal lines $(Y=0.25,0.5,0.75)$, for $\operatorname{Re}_{2}=100$ and $\lambda=-2,-6$, and -10 . It is seen that the rising absolute velocity ratios cause an increase of the maximum magnitude of velocity profiles inside the cavity. Moreover, the range of the maximum $U$ velocity $\left(\left|U_{\max }\right|\right)$ within the cavity is expanded from the bottom wall towards the top wall with the rising of absolute

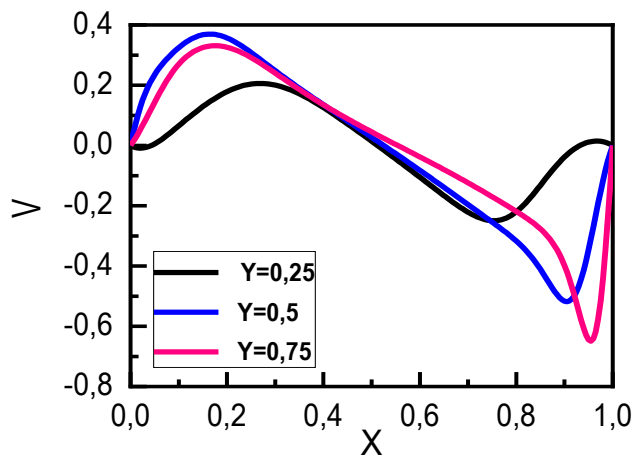

Fig. 11. Vertical velocity profile $V$ for $\lambda=-10$ through the horizontal lines $(Y=0.25, Y=0.5, Y=0.75)$.

velocity ratios (Figures 6, 8, 10). Whereas, the maximum $V$-velocity components $\left(\left|V_{\max }\right|\right)$ within the cavity is reached along $(Y=0.75)$ near the top right corner of the cavity (Figures $7,9,11)$.

\section{Conclusion}

An accurate second-order finite volume method has been presented to calculate steady two-dimensional flow in a two-sided lid-driven square cavity for antiparallel wall motion. Very accurate results of the asymmetrical flow driving $\left(R e_{1} \neq-R e_{2}\right)$ was conducted for $R e_{2}=100$ and $\lambda=-2$, 6 , and -10 . The evolution of the flow structure with the increased velocity ratios has been presented. In contrary to the conventional symmetrical flow driving with

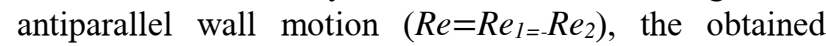
numerical results show that the flow pattern evolves asymmetrically with the appearance of two secondary vortices confined to the bottom right of the cavity. Fluid proprieties are directly and significantly affected when different velocity ratios cause incitement. Consequently, no further symmetrical flow exists about the centre of the cavity.

\section{References}

1. H.C. Kuhlmann, M. Wanschura, H.J. Rath, J. Fluid Mech, 336, 267 (1997).

2. H.C. Kuhlmann, M. Wanschura, H.J. Rath, Eur. J. Mech. B/Fluids. 17, 561 (1998).

3. S. Albensoeder, H.C. Kuhlmann, H.J. Rath, J. Theor. Comput. Fluid. Dyn, 14, 223 (2001).

4. A. Perumal, A.K. Dass, CFD. Lett, 2, 13 (2010).

5. A. Perumal, A.K. Dass, CFD. Lett, 2, 25 (2010).

6. A. Perumal, J. Appl. Sci. Thermodyn. Fluid. Mech, 6, 1 (2012).

7. A. Munir, M. Riswan, M. Khan, A. Salah. CFD. Lett, 5, 57 (2013).

8. K.T. Chen, C.C. Tsai, W.J. Luo, C.N. Chen, Theor. Comp. Fluid. Dyn, 27, 767 (2013).

9. K.T. Chen, C.C. Tsai, W.J. Luo, C.W. Lu, C.H. Chen, J. Mech, 31, 153 (2015).

10. S. Arun, A. Satheesh, J. Alexandria. Eng, 54, 795 (2015). 
11. T. Lemée, G. Kasperski, G. Labrosse, R. Narayanan, Comput. Fluids, 119, 204 (2015).

12. C. Prasad, A.K. Dass, Comput. Fluids, 140, 297 (2016).

13. A Perumal, TSEP, 6, 48 (2018).

14. N.AC. Sidic, S.A Razali, J. Appl. Mech. Math, 554, 675 (2014)

15. J.H. Ferziger, M. Peric', Computational Methods for Fluid Dynamics, in Computational Methods for Fluid Dynamics (Springer, Berlin/Heidelberg, New York, 157 (2002).

16. S.V. Patankar, D.B. Spalding, Int. J. Heat. Mass. Tran, 15, 1787 (1972).

17. U. Ghia, K.N. Ghia, C. Shin, J. Comput. Phys, 48, 387 (1982). 